
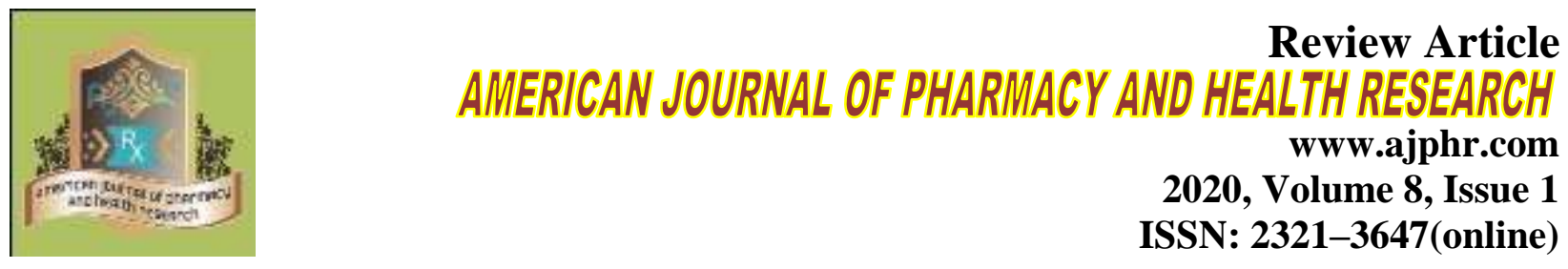

\title{
Self-Emulsifying Drug Delivery System (SEDDS): An Approach To Increase The Solubility Of Lipophilic Drugs
}

\author{
Sunitha Reddy M*, Sowmya V \\ Centre for Pharmaceutical Sciences, Institute of Science and Technology, JNTUH, Kukatpally, \\ Hyderabad, 500085, Telangana, India.
}

\begin{abstract}
Solubility of orally administered medication is the significance test for pharmaceutical industry as almost $40 \%$ of recently propelled medications have low water solubility which prompts their lower dissolution and bioavailability, bringing about high intra and inter subject changeability and absence of dose proportionality. This can be expanded by various techniques including salt formation, complex formation, solid dispersions. Self-emulsifying drug delivery System (SEDDS) is picking up fame for improving the solvency of lipophilic medications. Selfemulsifying drug delivery systems (SEDDS), which are isotropic blends of oils, surfactants, solvents and co-solvents/co-surfactants which have the novel capacity of forming fine oil-inwater $(\mathrm{o} / \mathrm{w})$ micro emulsions upon mild agitation pursued by dilution in aqueous media, i.e., GI fluids. It can be utilized for the formulations so as to improve the oral ingestion of profoundly poorly soluble drugs. Self- emulsifying drug delivery systems are composed of emulsion droplets whose size ranges from 50-500 nm whereas the systems which have droplet size below $50 \mathrm{~nm}$ are known as Self-Nano emulsifying drug delivery system (SNEDDS).
\end{abstract}

Keywords: Self- Emulsifying Drug Delivery System, Isotropic Mixtures, Gastrointestinal tract, surfactants, self-emulsification, bioavailability. 


\section{INTRODUCTION}

Oral route is the best way for chronic drug therapy, but drug dissolution is the rate limiting step for the water insoluble drugs. About $40 \%$ of the marketed formulations are lipophilic in nature which leads to decrease in bioavailability, intra and inter subject variability ${ }^{[1]}$. In order to overcome such problems there are various formulations such as liposomes, solid dispersions, salt formation, nanoparticles etc.

To increase the oral bioavailability of BCS Class- II drugs, lipid based formulations are preferred such as self-emulsifying drug delivery systems, self- micro emulsifying drug delivery system ,self-Nano emulsifying drug delivery system solutions or suspensions of the drug in lipid medium ${ }^{[2]}$.

Self-emulsifying drug delivery system is defined as a lipid based formulation consists of an isotropic mixtures of oils (natural or synthetic), surfactants (non-ionic surfactants) and cosolvents (hydrophilic). The droplet size of the formulation ranges from $200 \mathrm{~nm}-5 \mu \mathrm{m}$ and the dispersion appears turbid.

SEDDS are stable preparations which increases dissolution of the poorly soluble drugs, provided by a small particle size thus a large area of dispersion upon oral administration. These formulations form fine emulsions in the gastrointestinal tract upon mild agitation provided by gastric mobility and the partitioning of drug between oil and water phase which occurs due to large interfacial area thus increases solubility and increases absorption ${ }^{[1]}$.

\section{SELF-NANO EMULSIFYING DRUG DELIVERY SYSTEM:}

SNEDDS are considered as the Nano emulsions formed by the SEDDS. These are the isotropic mixtures of oils (synthetic), surfactants and co-surfactants which have the ability of forming fine Nano emulsion $(\mathrm{o} / \mathrm{w})$ under mild agitation followed aqueous media. These formulations are also called as Nano emulsion, mini emulsion, sub micro emulsion, ultra-fine emulsion ${ }^{[3]}$.

These are heterogeneous dispersions formed by two immiscible liquids ( $\mathrm{o} / \mathrm{w}$ or w/o) having mean droplet size ranging from 20nm-200nm(Nano metric scale) independent of method of preparation which is particularly important to increase solubility of lipophilic drugs (Atorvastatin, Simvastatin).

SNEDDS was formulated by using non-ionic surfactants, medium chain triglycerides for better oral ingestion. These formulations are important for the increase of oral absorption of the drugs as well as the plasma profile of drug concentration.

Types of Nano emulsions ${ }^{[3]}$ : 
1) Oil in water Nano emulsion:

Dispersed phase: water

Continuous phase: oil

2) Water in oil Nano emulsion:

Dispersed phase: water

Continuous phase: oil

3) Bi continuous Nano emulsion:

In this type of emulsion surfactant is soluble in both oil and water phase, and droplet disperse in both oil and water phase.

\section{SELF MICRO EMULSIFYING DRUG DELIVERY SYSTEM:}

These are micro emulsions defined as an isotropic mixtures of oils (natural or synthetic), surfactants (solid or liquid) which have the ability of forming fine micro emulsion $(\mathrm{o} / \mathrm{w})$ under mild agitation followed by dilution in aqueous media ${ }^{[4]}$. Micro emulsion can be achieved by chemical rather than mechanical means i.e., by intrinsic property of the drug formulation rather than by special mixing and handling.

SMEDDS formulation have the particle size ranging from $1 \mathrm{~nm}-100 \mathrm{~nm}$ and gives transparent or bluish appearance. SMEDDS are important to increase bioavailability of poorly water soluble drugs was reported by Pouton ${ }^{[5]}$ in 1982. After self-dispersion, the drug is distributed throughout gastrointestinal tract as fine droplets. The stability of formulation of SMEDDS i.e., region of micro emulsion can be identified by constructing the pseudo ternary phase diagram of selected excipients using water titration method. (Table No.1)

\section{Advantages of SEDDS over conventional drug delivery systems:}

1. Reduced particle size leads to enhanced bioavailability.

2. Reproducible blood time profiles can be obtained.

3. Selective drug targeting and drug protection from the hostile environment in gut.

4. Ease of manufacturing and scale up ${ }^{[1]}$.

5. Minimizes gastric irritation as oil droplets easily passes and facilitates wide distribution of drug throughout the gastro intestinal tract ${ }^{[1]}$.

6. Protection of sensitive drug substances ${ }^{[7]}$.

7. Reduced variability including food effects.

\section{Disadvantages:}

1. Lack of good predictive in-vitro models for the assessment of the formulations ${ }^{[1]}$.

2. Chemical instability of drugs. 
3. High concentration of surfactants in the formulation (approximately 30-60\%) which irritates gastrointestinal tract ${ }^{[7]}$.

4. Traditional dissolution methods do not work because these formulations primarily depends on digestion prior to drug release.

5. Usage of volatile co-solvents in the formulation are known to migrate in to the shells of hard or soft gelatin capsules which leads to precipitation of water insoluble drugs ${ }^{[7]}$. FORMULATION OF SEDDS:

The fundamental point of SEDDS is to build the solubility of poorly water soluble drugs i.e., BCS class- II drugs (Eg: Ketoconazole, Ibuprofen, Nifedipine etc). The excipients utilized in the definition of SEDDS are fundamentally founded on solubility. SEDDS can be formulated by the admixture of API along with excipients include oils, surfactants, co-solvents etc.

\section{Active pharmaceutical ingredient:}

Drugs with high permeability and low solubility are preferred to increase their solubility Eg: Danazol, Piroxicam, Ketoprofen, Diclofenac ${ }^{[1]}$.

\section{Excipients:}

Determination of excipients is basic in the detailing of SEDDS in considering the pharmaceutical acceptability and toxicity issues. Self-emulsification is a procedure which is seen at a specific proportions of oils to surfactant, surfactant to co-surfactant ratio and temperature must be considered during the determination of excipients ${ }^{[10]}$.

\section{Oils:}

Oils are considered to be one of the essential excipient in the formulation of sedds. It helps in solubilizing the lipophilic part of drug and facilitates the self-emulsification process ${ }^{[9]}$. It increases the transport of lipophilic part of the drug via the intestinal lymphatic system thus increases the absorption from GIT. The rate of absorption depends on the molecular weight of triglycerides. Both long chain and medium chain triglyceride oils with different degrees of saturation are used in the formulation of SEDDS ${ }^{[10]}$. Medium chain triglycerides are ampiphilic compounds and have more solubility and mobility in the lipid/water interface. When long chain triglycerides are used, Cremophor RH40 which favors the formation of micro emulsion is used in large concentration. Types of oils used in formulation of SEDDS include corn oil, peanut oil, soya bean oil, sesame oil Etc.

\section{Surfactants:}


Several compounds exhibits properties of surfactants are utilized for the framework of selfemulsifying systems, yet the decision is restricted as not many surfactants are orally worthy. The most broadly used are non-ionic surfactants with a relatively high hydrophilic lipophilic balance (HLB). Safety is the major deciding component in picking a surfactant ${ }^{[10]}$.

Emulsifiers of natural source are favored than the manufactured surfactant, however they have a constrained self-emulsification limit. There is a relationship between the size of droplet and the concentration of surfactants being utilized. Increase in the concentration of surfactants leads to reduction in the mean droplet size. This could be clarified by the adjustment of oil drops because of the localization of the surfactant at oil-water interface. Then again, increase in the surfactant concentration may increase the mean droplet size. This phenomenon could be ascribed to the interfacial disturbance evoked by increased water penetration in to the oil droplets mediated by the increase in the surfactant concentration which leads to the release of oil droplets in to water phase ${ }^{[10]}$. The surfactants utilized in the formulation are known to improve the bioavailability by various mechanisms including increase in drug dissolution, expanded intestinal epithelial permeability, increase in penetration through tight junctions and constrained p-glycoprotein drug efflux. However, the huge amount of surfactant may cause moderate reversible changes in intestinal wall permeability or may bother GIT. Non- ionic surfactants used in formulation of SEDDS includes tween $80^{[9]}$, span 80 , cremophor RH40 etc.

\section{Co-solvents:}

The generation of an ideal SEDDS requires moderately high concentrations (>30\%) of surfactants, thus the concentration of surfactant can be reduced by fuse of co-surfactant. Role of co-surfactant together with surfactant is to bring down the interfacial tension to an extremely little even transient negative value ${ }^{[10]}$. At this worth the interface would expand to form fine dispersed droplets and along with it, it absorbs more surfactant and surfactant/co-surfactant until their bulk condition is drained enough to make interfacial strain positive again. However the utilization of co-surfactant in SEDDS is not required for most of non-ionic surfactants. The choice of surfactant and co-surfactant is vital not exclusively to the development of SEDDS, yet additionally to solubilization of the formulation in the SEDDS.

Commonly used co-solvents in the formulation of SEDDS are polyethylene glycol [9], ethanol, glycerin, polypropylene glycol, polyoxyethylene etc. to dissolve hydrophobic drugs in the lipid.

\section{Additives:}

\section{Anti-oxidants:}


Lipophilic anti-oxidants such as a-tocopherol, p-carotene, butylated hydroxytoluene (BHT), butylated hydroxyanisole (BHA), ascorbic palmitate and propyl gallate are used to overcome the oxidation of excipients in SEDDS formulation ${ }^{[9]}$.

\section{Polymers:}

In numerous cases, the dissolvability limit of SEDDS is lost which causes drug precipitation. Water soluble polymers such as hydroxyl propyl methylcellulose (HPMC) ${ }^{[1]}$, poly ethylene glycol (PEG) 4000, poly vinyl pyrrolidone (PVP) are used to overcome precipitation to form supersaturable self-emulsifying system. Adsorption of the polymer on to the crystal surface of the drug through hydrogen bonding plays a major role in the inhibition of crystallization and precipitation.

\section{MECHANISM OF SELF EMULSIFICATION:}

The mechanism of self -emulsification has not yet been elucidated. According to Reiss the selfemulsification occurs when the change in entropy favoring dispersion is greater than the energy that is required to increase the surface area of dispersion. The free energy of a conventional emulsion formulation is a direct function of the energy that is required to create a new surface between the oil and water phases. Thermodynamic relationship for the net free energy change is given by the equation ${ }^{[10]}$ :

$\Delta \mathrm{G}=\sum \mathrm{Ni} \pi \mathrm{r} 2 \sigma$

$\Delta \mathrm{G}=$ free energy associated with the process

$\mathrm{Ni}=$ number of droplets

$r=$ radius of the droplets

$\sigma=$ interfacial energy

Both the phases of emulsion tends to change with time to reduce the interfacial area and free energy of the system. Conventional emulsifiers stabilizes emulsions resulting from aqueous dilution by forming a layer around the droplets of emulsion, decreasing the interfacial energy and forms a barrier to mixture ${ }^{[11]}$. Emulsification also occurs spontaneously with SEDDS as the free energy required either low or negative or positive. For emulsification to takes place it is important that the interfacial structure should show no resistance against surface shearing. The interface frames among water and oil stages upon the addition of double mixture (oil/non-ionic surfactant to water). This is trailed by solubilization inside oil phase then the aqueous penetration happens through interface. It occurs until the solubilization limit reaches close to the interface. Dispersed LC (liquid crystalline) phase can be seen upon further aqueous penetration. 
Table 1: Difference Between SMEDDS And SNEDDS [ 6 ]

\begin{tabular}{|c|c|c|}
\hline Features & SMEDDS & SNEDDS \\
\hline & $\begin{array}{l}\text { Self-micro emulsifying drug delivery } \\
\text { system }\end{array}$ & $\begin{array}{l}\text { Self-Nano emulsifying drug } \\
\text { delivery system }\end{array}$ \\
\hline Globule size & $100-200 \mathrm{~nm}$ & $50-100 \mathrm{~nm}$ \\
\hline Appearance & Optically clear to translucent & Optically clear \\
\hline Stability & Thermodynamically stable & $\begin{array}{l}\text { Thermodynamically unstable } \\
\text { and kinetically stable }\end{array}$ \\
\hline Order of mixing & $\begin{array}{l}\text { Surfactant is mixed with oil phase } \\
\text { followed by titration of the resultant } \\
\text { mixture with the aqueous phase }\end{array}$ & No effect \\
\hline Concentration of oil & $<20 \%$ & As less as possible \\
\hline Energy required & $\begin{array}{l}\text { High energy is required for the } \\
\text { formulation compared to SNEDDS }\end{array}$ & $\begin{array}{l}\text { Less energy is required in } \\
\text { formulation }\end{array}$ \\
\hline Type of system & $\begin{array}{l}\text { Equilibrium system (micro emulsion } \\
\text { system is mono phasic with swollen } \\
\text { micelles }\end{array}$ & $\begin{array}{l}\text { Nano emulsion is true } \\
\text { emulsion i.e., non-equilibrium } \\
\text { system which has the } \\
\text { tendency to separate in to } \\
\text { constituent phases }\end{array}$ \\
\hline
\end{tabular}

\section{CONSTRUCTION OF PSEUDO-TERNARY PHASE DIAGRAMS:}

To determine the ideal concentration of oil, surfactant, co-surfactant phase diagrams are developed ${ }^{[12]}$. The formulations were set up by blending surfactant and co-surfactant in proportions 1:1, 1:2, 1:3 (Smix,w/w). Ternary blends with varying compositions of Smix and oil were prepared. Nine distinct blends of oil and Smix 1:9, 2:8, 3:7, 4:6, 5:5, 6:4, 7:3, 8:2, and 9:1, were made so that the greatest ratios were covered to characterize the limit of phase formed in the phase diagrams ${ }^{[15]}$.

EVALUATION OF SEDDS:

Various tests are performed for the characterization and evaluation of SEDDS.

\section{Drug content:}

Drug from the pre-weighed SEDDS is extracted by making it soluble in a suitable solvent. The obtained extract is analyzed by appropriate analytical method ${ }^{[1]}$.

\section{Dispersibility test:}

This test is carried out to evaluate the capacity to scatter into emulsion and classify the size of resultant globules. It is conveyed by utilizing a standard USP dissolution apparatus II (paddle type). One $\mathrm{ml}$ of every formulation is added to $500 \mathrm{ml}$ of water at $37+0.5^{\circ} \mathrm{C}$ and paddle is turned at 50rpm ${ }^{[10]}$. On titration with water SEDDS formulation forms a blend or gel which is of various sort depending upon which the in-vitro exhibition of formulation can be evaluated utilizing the following grading system: 
Grade A: Rapidly forming (within $1 \mathrm{~min}$ ) Nano emulsion, having a reasonable or somewhat bluish appearance.

Grade B: Rapidly forming, somewhat less clear emulsion, having a pale bluish white appearance.

Grade C: Fine smooth emulsion that formed within $2 \mathrm{~min}$.

Grade D: Dull, grayish white emulsion having somewhat slick appearance that is delayed to emulsify (longer than $2 \mathrm{~min}$ ).

Grade E: Formulation, exhibiting either poor or negligible emulsification with enormous oil globules present on the surface.

Evaluation of Grade A and Grade B formulations will stay as Nano emulsion when dispersed in GIT. While formulation falling in Grade C could be prescribe for SEDDS formulation.

\section{Rheological properties:}

The SEDDS system can also be administered in soft gelatin capsules, where it should have considerable flow properties for handling. The rheological properties (thickness, flow, thixotropy, static yield, creep esteem) of formulation (diluted to $5 \% \mathrm{v} / \mathrm{v}$ water) are dictated by rotational viscometers, computerized instruments combined with either cup and bob or coaxial estimating device ${ }^{[11]}$. A sort of rotational viscometer has additionally been utilized for assurance of thickness of fresh as well as different SEDDS formulations which has been stored for longer term of time. Determination of thickness of fluid SEDDS additionally demonstrates whether the system is o/w or w/o, as low consistency systems are o/w and high consistency systems are typically w/o in nature. Consistency of formulation is indirect proportional to dilution ${ }^{[13]}$.

\section{Examination of thermodynamic stability:}

The physical strength of a formulation is significant for its exhibition as it can be unfavorably influenced by precipitation of the medication in excipient matrix. Poor physical dependability of the formulation can lead to phase partition of excipients which influences bioavailability as well as viability. Additionally, incompatibilities among the formulation and gelatin shell of capsule (capsule filled formulation) may cause fragility, softness, delay in disintegration time or incomplete drug release. The following cycles are carried out to examine them.

- Heating Cooling Cycle: Six cycles of cooling and warming between refrigeration temperature $\left(4^{\circ} \mathrm{C}\right)$ and raised temperature $\left(45^{\circ} \mathrm{C}\right)$ with exposure at every temperature for at the very least 48 hours are carried ${ }^{[10]}$. Those formulations, which are steady, are then subjected to centrifugation test. 
- Test for Centrifugation: Formulations which pass the heating cooling cycle are centrifuged at $3500 \mathrm{rpm}$ for $30 \mathrm{~min}{ }^{[14]}$. Those formulations that don't demonstrate any phase partition are taken for the freeze thaw stress test.

- Freeze Thaw Stress Cycle: Three freeze thaw cycles b/w $-21^{\circ} \mathrm{C} \& 25^{\circ} \mathrm{C}$ with capacity at each temperature for not less than those formulations which pass this test show good stability with no phase partition, breaking or creaming. The formulations that pass this test are then additionally taken for dispersibility test for appraisal of self-emulsification effectiveness.

\section{Robustness to dilution:}

Emulsions upon dilution with various dissolution media ought not to demonstrate any stage partitions or precipitation of medication even after $12 \mathrm{hrs}$ of capacity, such detailing is considered as powerful to dilution ${ }^{[13]}$.

\section{Turbidimetric evaluation:}

Turbidity is a parameter for assurance of droplet size and self-emulsification time. Fixed amount of SEDDS is added to fixed amount of appropriate medium (0.1 N HCL or Phosphate Buffer) under ceaseless mixing at $50 \mathrm{rpm}$ on magnetic stirrer at ideal temperature and the turbidity is estimated utilizing a turbid meter ${ }^{[13]}$. Since the time required for complete emulsification is excessively short, it is unimaginable to monitor the rate of change of turbidity for example pace of emulsification. Turbidimetric assessment is carried out to monitor the development of droplet after emulsification.

\section{Particle size and droplet size analysis:}

Dynamic light scattering (DLS) or Photon correlation spectroscopy (PCS) or Laser Diffraction Techniques are utilized to determine droplet size of emulsion ${ }^{[11]}$. Various types of equipment's are accessible for estimation of molecule size includes Molecule Size Analyzer, Mastersizer, Zeta sizer and so forth which can gauge measures somewhere in the range of 10 and $5000 \mathrm{~nm}^{[1]}$.

\section{Liquefaction time:}

It is proposed to appraise the time required by solid SEDDS to liquefy in-vivo in the absence of agitation to simulated GI conditions ${ }^{[13]}$.

\section{Determination of refractive index:}

Refractive Index and percent transmittance are resolved to check the transparency of definition. Refractive index can be determined by placing a drop of solution on the slide and then contrast with water $($ R.I $=1.333)$. Refractive Index of the formulation is estimated by refractometer. The percent transmittance of the formulation is estimated at a specific wavelength utilizing UV 
spectrophotometer by utilizing refined water as blank. If Refractive index of definition is like that of water and formulation having percent transmittance is more prominent than $99 \%$, at that point the formulation are transparent in nature ${ }^{[13]}$.

\section{In-vitro diffusion study:}

This investigation is done to determine drug release behavior utilizing dialysis technique where phosphate buffer ( $\mathrm{pH}$ 6.8) is commonly used as dialyzing medium. One part of the arrangement layer is tied with a string and $1 \mathrm{ml}$ of the SEDDS formulation along with $0.5 \mathrm{ml}$ of dialyzing medium are filled in the film ${ }^{[1]}$. The opposite part of the arrangement likewise tied with string and then allowed to rotate in dialyzing medium at 100rpm utilizing dissolution apparatus. Samples are pulled back at various time intervals and dilutions are analyzed. Sample volumes are withdrawn periodically and are replaced with fresh dialyzing medium ${ }^{[11]}$.

\section{Self-emulsification time:}

The self-emulsification time is controlled by utilizing USP dissolution device 2 at $50 \mathrm{rpm}$, where $0.5 \mathrm{~g}$ of SEDDS formulations is introduced into $250 \mathrm{ml}$ of $0.1 \mathrm{~N} \mathrm{HCL}$ or $0.5 \%$ SLS (Sodium Lauryl Sulfate) solution ${ }^{[15]}$. The ideal opportunity for emulsification at room temperature is shown as self-emulsification time for the formulation.

\section{In-vitro dissolution technique:}

The quantitative in vitro dissolution studies are carried out to determine the drug release from oil phase into aqueous phase by USP-Type II dissolution apparatus utilizing $500 \mathrm{ml}$ of simulated gastric liquid containing $0.5 \% \mathrm{w} / \mathrm{v}$ of SLS (sodium lauryl sulphate) at $50 \mathrm{rpm}$ and temperature at $37 \pm 0.5^{\circ} \mathrm{C}^{[15]}$. Samples are withdrawn periodically at regular intervals of time and are replaced with fresh medium. Samples are collected and analyzed by utilizing UV spectrophotometer or some other appropriate method ${ }^{[16]}$.

\section{CONCLUSION:}

SEDDS are a promising methodology for medications with poor water solubility and subsequently helpful for BCS Class II and IV drugs upon administration. When the SEDDS formulation reaches GIT it absorbs water from the surroundings and forms an emulsion (oil in water type) which disperses and forms in to fine droplets that provides high surface area for the drug to penetrate in to the surrounding medium. SEDDS are formulated as liquid dosage forms but solid dosage forms are more preferred due to its ease of handling, better transportation and stability. With the future advancement of this innovation, SEDDS will keep on empowering 
novel applications in drug delivery and solves the issues related with the delivery of lipophilic drugs.

\section{REFERENCES:}

1. Kshitija Khedekar And Swati Mittal, Self Emulsifying Drug Delivery System (Sedds): A Review, International Journal Of Pharmaceutical Sciences And Research, Projected Impact Factor (2018): 0.83, Cite Score (2017): 0.27, 2013; 4494-4507.

2. Anayatollah Salimi, Behzad Sharif Makhmal Zadeh, Ali Asghar Hemati, And Sanaz Akbari Birgani, Design And Evaluation Of Self-Emulsifying Drug Delivery System (Sedds) Of Carvedilol To Improve The Oral Absorption, Jundishapur Journal Of natural Pharmaceutical Products, 2014; 9(3): E16125.

3. Sagar K. Savale, A Review - Self Nanoemulsifying Drug Delivery System (Snedds), International Journal Of Research In Pharmaceutical And Nano Sciences, 2015; 4(6): 385 - 397.

4. Shraddha D. Pawar, Nayan A. Gujarathi, Bhushan R. Rane, Sunil P. Pawar, Self-Micro Emulsifying Drug Delivery System (Smedds): A Promising Drug Delivery System For Enhancement Of Bioavailability, Indian Journal Of Drugs, 2016; 4(3): 90-108.

5. Yeole Manali, Dhole Shashikanth, Kulkarni Nilesh, Development And Evaluation Of Poorly Aqueous Soluble Drug Racecadotril By Using Solid Self Microemulsifying Drug Delivery Approach, International Research Journal Of Pharmacy, 2014; 5(7): 565-575.

6. Mittal Tanya And Kumar Hari S.L, Smedds/Snedds: An Emerging Technique To Solubility Enhancement For The Pharmaceutical Industry, World Journal Of Pharmacy And Pharmaceutical Sciences, 2017; 6(7):317-336.

7. Patel Vipul P, Desai Tushar R, Kapupara Pankaj P, Atara Samir A, Keraliya Rajesh A, Self Emulsifying Drug Delivery System: A Conventional And Alternative Approach To Improve Oral Bioavailability Of Lipophilic Drugs, International Journal Of Drug Development And Research, 2010.

8. Bhupinder Singh, Shantanu Bandopadhyay, Rishi Kapil, Ramandeep Singh, \& O.P. Katare, Self Emulsifying Drug Delivery System (Sedds): Formulation Development, Characterization, And Applications, Critical Reviews ${ }^{\mathrm{TM}}$ In Therapeutic Drug Carrier Systems, 2009; 26(5): 427-521.

9. Anjan Kumar Mahapatra, P. Narasimha Murthy, B. Swadeep, Ranjit Prasad Swain, SelfEmulsifying Drug Delivery Systems (Sedds): An Update From Formulation 
Development To Therapeutic Strategies, International Journal Of Pharmtech Research, 2014; 6(2): 546-568.

10. Pallavi M. Nigade, Swapnil L. Patil, Shradha S. Tiwari, Self Emulsifying Drug Delivery System (Sedds): A Review, International Journal Of Pharmacy And Biological Sciences, 2012; 2(2): 42-52.

11. Patel P A, Et Al: Self Emulsifying Drug Delivery System: A Review. Research Journal Of Pharmacy And Technology 2008; 1(4): 313-323.

12. Nagaraja Sreeharsha, Arshia Shariff1, Yogesh A Shendkar, Bandar E. Al-Dhubiab1, Girish Meravanige, Development And Evaluation Of A (Sedds) Self emulsifying Drug Delivery System For Darifenacin Hydrobromide, Indian Journal Of Pharmaceutical Education And Research,53(2), 2019;S204-S212.

13. Suresh Chandra Pandey, Rajveer Kaur, Gangadharapp H.V., Sachin J., Self- Emulsifying Drug Delivery System (Sedds): A Review, World Journal Of Pharmacy And Pharmaceutical Sciences, 2018; 7(6) :494-515.

14. S. Shafiq, F. Shakeel, S. Talegaunker, Fj Ahmed, R.K. Khar, M. Ali., Development And Bioavailabilityassessment Of Ramipril nanoemulsion Formulation, Eur. J. Pharm. Biopharm, 2007; 66(2): 227-43.

15. Sumandeep Vidyapeeth, Preparation And Characterization Of Sedds, Chapter 7, 117-144.

16. Baddam Sunitha Reddy, G. Harish And Md. Fazal Ul-Haq, Formulation And In-Vitro Characterization Of Solid - Self Nanoemulsifying Drug Delivery System (S-Snedds) Of Rilpivirine, International Journal Of Pharmaceutical Sciences And Research, 2016; 7(7): 3117-3129.

\section{AJPHR is}

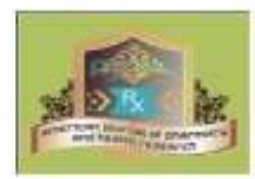

Peer-reviewed monthly

Rapid publication

Submit your next manuscript at editor@ajphr.com / editor.ajphr@gmail.com 